\title{
Sliding Mode Output Feedback Control for a Class of Uncertain Discrete-time Markov Jump Systems
}

\author{
Fengzhi Huang*, Yan Hou, Shijie Zhang and Qingsheng Shi \\ College of Electrical Engineering, Henan University of Technology, Zhengzhou, Henan Province, China \\ *Corresponding author
}

\begin{abstract}
This paper considers the development of output feedback sliding mode controllers for a class of discrete-time Markov jump systems. The uncertainty is matched. A static output feedback control law is produced when considering the system satisfied the 'Kimura-Davison' condition. If the condition is not satisfied, a dynamic output feedback control law is produced.
\end{abstract}

Keywords-MARKOV jump systems; sliding mode control; uncertainty; output feedback

\section{INTRODUCTION}

A large class of physical systems have more than one structure, because they may suffer random changes which caused by abrupt phenomena as parameter shifting, component and interconnection failures and so on. Systems with such character may be modeled as hybrid systems. One class of hybrid system, Markov jump systems, in which the modeprocess is a continuous-time discrete-state Markov process taking values in a finite set, have been extensively studied in the past decades, and some good results have been got.

In another active research area, the sliding mode control has attractive features to keep systems insensitive to the uncertainties on the sliding surface. The salient advantages of sliding control are:1) fast response and good transient performance; 2) robustness against a class of model uncertainties or perturbations. To our best knowledge, a great amount of work has been conducted on Markov jump systems, such as $\mathrm{H}_{2}, \mathrm{H}_{\infty}$ and mixed $\mathrm{H}_{2} / \mathrm{H}_{\infty}$ control or filters for Markov jump systems with known and unknown noise statics with either LQ or $\mathrm{H}_{\infty}$ performances, the design of controller or filter are mostly based on the assumption of the states of the system are completely known. However, in practice, for some reason the states are hard to be measured completely or may costly to be measured, on the other side, the output of the system are easily measurable, so in this paper, for a class of discrete-time Markov jump system, we consider to design a controller that based on the output feedback signals.

In this paper, we consider the problem of a class of discrete-time Markov jump systems with matched uncertainties, which are bounded by a function related to the output signals. When the system satisfy the pole placement conditions relating to controllable and observability and also the well known 'Kimura-Davison' condition pertaining to the system dimensions, a static sliding mode output feedback controller is proposed. Otherwise if the condition, which is hard assumption, is not satisfied, a dynamic sliding mode output feedback control strategy is procedure.

\section{SySTEM DESCRIPTION AND PROBLEM FORMULATION}

The systems we considered is a class of uncertain discretetime Markov jump linear systems in a fixed probability space $(\Omega, \mathrm{F}, \mathrm{P})$ as.

$$
\left\{\begin{array}{l}
x(k+1)=A\left(\theta_{k}\right) x(k)+B\left(\theta_{k}\right)[u(k)+F(u, x, k)] \\
y(k)=C\left(\theta_{k}\right) x(k)
\end{array}\right.
$$

where $x(k)$ is the state vector, $u(k) \in \mathcal{R}^{m}$ is the control input, $F(u, x, k)$ is the uncertain and $\omega \in \mathcal{R}^{l}$ is the disturbance, $y(k) \in \mathcal{R}^{q}$ is the measurement output. $\left\{\theta_{k}, k \geq 0\right\}$ is a discrete-time homogeneous Markov chain, taking value in a finite set of $\sigma=\{1, \ldots, N\}$, with a transition probabilities matrix $\Lambda=p_{i j}$

$$
\lambda_{i j}=\operatorname{Pr}\left(\theta_{k+1}=j \mid \theta_{k}=i\right)
$$

where $\lambda_{i j} \geq 0, \forall i, j \in \sigma$ and $\sum_{j=1}^{N} \lambda_{i j}=1$

For each possible value of $\theta_{k}=i, i \in \sigma$, we will denote $A\left(\theta_{k}\right), B\left(\theta_{k}\right), C\left(\theta_{k}\right)$ associated with mode $i$ as $A_{i}, B_{i}, C_{i}$.

For the system (1), we assume that the following assumptions are satisfied:

1) The matrices $A_{i}, B_{i}, C_{i}$ re constant matrices with appropriate dimensions;

2) The matrices $B_{i}, C_{i}$ are full rank and $m \leq q<n$;

3) The uncertain function $F(u, x, k)$ is bounded as

$$
\|F(u, x, k)\| \leq \alpha\|u\|+\beta(y, k)
$$

where $\alpha$ is a known scalar such that $0<\alpha<1$, and $\beta(y, k)$ is a known function. 


\section{SLIDING Mode OUtPut FeEdBack CONTROL STRATEGIES FOR MJS}

In this part, two control strategies based on the output feedback information for MJS are designed. The first controller is constructed based on the system output information directly; The other one is constructed both with system state information which could be measured and output information.

\section{A. Static Output Feedback Control Design For MJS}

The main problems when we use the sliding mode method to construct a controller are to consider possible construction of a switching surface based on static output measurements:

$$
S=G_{i} y(k)=0
$$

where $G_{i}$ is needed to be selected.

In order to get a regular form of system (1), we can choose a nonsingular matrix $T\left(\theta_{k}\right)$, for each possible value $\theta_{k}=i, i \in \sigma$ denoted as $T_{i}$, by transforming system state $z(k)=T_{i} x(k)$, system (2) could be changed as following:

$$
z(k+1)=\tilde{A}\left(\theta_{k}\right) z(k)+\tilde{B}\left(\theta_{k}\right)[u(k)+F(u, x, k)]
$$

denote $\tilde{A}\left(\theta_{k}\right), \tilde{B}\left(\theta_{k}\right)$ as $\tilde{A}_{i}, \tilde{B}_{i}$ respectively for each possible $\theta_{k}=i, i \in \sigma$ then (5) could be written as

$$
\left\{\begin{array}{l}
z(k+1)=\tilde{A}_{i} z(k)+\tilde{B}_{i}[u(k)+F(u, x, k)] \\
y(k)=\tilde{C}_{i} z(k)
\end{array}\right.
$$

where $\tilde{A}_{i}=T_{i}^{-1} A_{i} T_{i}, \tilde{B}_{i}=T_{i} B_{i}=\left[\begin{array}{c}0 \\ B_{2 i}\end{array}\right], \tilde{C}_{i}=C_{i} T_{i}^{-1}, \tilde{B}_{2 i} \in \mathcal{R}^{m}$

Without loss of generation, we assume that $z(k), A_{t}$ have the following form

$$
z(k)=\left[\begin{array}{c}
z_{1}(k) \\
z_{2}(k)
\end{array}\right], \tilde{A}_{i}=\left[\begin{array}{cc}
\tilde{A}_{11 i} & \tilde{A}_{12 i} \\
\tilde{A}_{21 i} & \tilde{A}_{22 i}
\end{array}\right]
$$

and $z_{1}(k) \in \mathcal{R}^{n-m}, z_{2}(k) \in \mathcal{R}^{m}$, such that (7) could be written as

$$
\begin{gathered}
z_{1}(k+1)=\tilde{A}_{11 i} z_{1}(k)+\tilde{A}_{12 i} z_{2}(k) \\
z_{2}(k+1)=\tilde{A}_{21 i} z_{1}(k)+\tilde{A}_{22 i} z_{2}(k)+\tilde{B}_{2 i}[u(k)+F(u, x, k)]
\end{gathered}
$$

The static sliding mode (4) could be calculated as

$$
S=G_{i} y(k)=G_{i} \tilde{C}_{i} z(k)=\left[\begin{array}{ll}
\tilde{G}_{1 i} & \tilde{G}_{2 i}
\end{array}\right]\left[\begin{array}{l}
z_{1}(k) \\
z_{2}(k)
\end{array}\right]=0
$$

then we obtain

$$
z_{2}(k)=-\tilde{G}_{2 i}^{-1} \tilde{G}_{1 i} z_{1}(k)
$$

Substitute (11) into (7) we could get the ideal sliding mode equation

$$
\begin{gathered}
Z_{1}(k+1)=\hat{A}_{1} z_{1}(k) \\
S=\left[\begin{array}{ll}
\tilde{G}_{1 i} & \tilde{G}_{2 i}
\end{array}\right] z(k)=0
\end{gathered}
$$

where $\hat{A}_{i}=\tilde{A}_{11 i}-\tilde{A}_{12 i} K_{i}, K_{i}=\tilde{G}_{2 i}^{-1} \tilde{G}_{1 i}$ which are needed to be calculated.

Definition1 System (12) is said to be stochastically stable if, for every initial condition $z_{0} \in \mathcal{R}^{n-m}$ and $\theta_{0} \in \sigma$, the following holds

$$
E\left\{\sum_{k=0}^{\infty}\|z(k)\|^{2} \mid z_{0}, \theta_{0}\right\}<\infty
$$

where $E($.$) is mathematical expectation.$

Lemma1(Costa el al.,2005) When consider $\omega(k) \equiv 0$ the unforced system (2) is stochastically stable if and only if there exist a set of symmetric and positive definite matrices $P_{i}, i \in \sigma$ satisfying the following inequations

$$
A_{i}^{T} Q_{i} A_{i}-P_{i}<0
$$

where $Q_{i}=\sum_{j \in \sigma} p_{i j} P_{j}$.

Theorem 1 The reduced system (12)is stochastically stable if there exist a set of positive matrices $X_{i}, i \in \sigma$ and matrices $Y_{i}, \forall i \in \sigma$ such that the following LMI satisfied

$$
\left[\begin{array}{cc}
-\chi_{j} & \pi_{i}\left(\tilde{A}_{11 i} X_{i}-\tilde{A}_{12 i} Y_{i}\right) \\
* & -X_{i}
\end{array}\right]<0
$$

where

$$
\chi_{j}=\operatorname{diag}\left\{X_{1}, X_{2}, \cdots, X_{N}\right\}
$$

$$
\pi_{i}=\left[\sqrt{p_{i 1}} I, \cdots, \sqrt{p_{i N}} I\right]^{T}
$$

and the sliding mode parameter could be calculated by 


$$
K_{i}=Y_{i} X_{i}^{-1}
$$

Proof: Choose a stochastic Lyapunov function as

$$
V(z(k), k)=z_{1}^{T}(k) P_{i} z_{1}(k), \forall i \in \sigma
$$

Based on the definition 1 , for $\theta_{k}=i, \theta_{k+1}=j$,we could have

$$
\begin{aligned}
& E(\Delta(V))=E\left(V\left(z_{1}(k+1), j \mid z_{1}(k), i\right)-V\left(z_{1}(k), i\right)\right) \\
& \quad=z_{1}(k+1)^{T}\left(\sum_{j=1}^{N} p_{i j} P_{j}\right) z_{1}(k+1)-z_{1}(k)^{T} P_{i} z_{1}(k) \\
& =z_{1}(k)^{T}\left(\hat{A}_{i}^{T} Q_{i} \hat{A}_{i}-P_{i}\right) z_{1}(k)
\end{aligned}
$$

According to the Lemma 1 we could get if the following in-equation

$$
\hat{A}_{i}^{T} Q_{i} \hat{A}_{i}-P_{i}<0
$$

is satisfied then(12)is stochastically stable. Applying Schur complement to (25), we could get

$$
\left[\begin{array}{ccccc}
-P_{1}^{-1} & 0 & \cdots & 0 & \sqrt{p_{i 1}} P_{1} \hat{A}_{i} \\
* & -P_{2}^{-1} & & \vdots & \sqrt{p_{i 2}} P_{2} \hat{A}_{i} \\
* & * & \ddots & 0 & \vdots \\
* & * & * & -P_{N}^{-1} & \sqrt{p_{i N}} P_{N} \hat{A}_{i} \\
* & * & * & * & -P_{i}
\end{array}\right]<0
$$

where*represents for the symmetric part. Setting $X_{i}^{-1}=P_{i}$ performing a congruence transformation to (24) by $\operatorname{diag}\left\{I, X_{i}\right\}$ and denote $Y_{i}=K_{i} X_{i}$ we could get (17).

Therefore if (17) holds, the reduced order system (12) is stochastically stable and the desired sliding mode parameter could be calculated by equation (20). This completes the proof.

After designing the sliding mode, a sliding mode control law need to be designed to drive the system states onto the sliding mode.

Theorem 2 Assume the condition in Theorem 1 holds, and the sliding surface (4) is calculated, if the following inequalities hold,

$$
\begin{gathered}
(1-q T)^{2} Q_{i}-P_{i}<0 \\
\gamma\left\|\tilde{G}_{i} \tilde{B}_{i}\right\|-2(1-q T)\|S(k)\|<0
\end{gathered}
$$

Then the following control law makes the ideal sliding mode stochastically stable, and the system (7) will be stochastically stabilized.

Using the discrete sliding convergence law,

$$
S(k+1)-S(k)=-\epsilon \operatorname{sign}(S(k))-q T s(k)
$$

the sliding mode control law is designed as

$$
\begin{gathered}
u=-\left[\tilde{G}_{i} \tilde{B}_{i}\right]^{-1}[\bar{G} y(k)-(1-q T) S(k)+\epsilon \operatorname{Tsign}(S(k))]+\bar{u}_{1} \\
\bar{u}_{1}=-\left\{\frac{\alpha[\| \bar{G}-(1-q T)) y(k) \|]+\beta(y, k)}{1-\alpha}\right\} \frac{S}{\|S\|}
\end{gathered}
$$

where $\bar{G}=\tilde{G}_{i} \tilde{A}_{i} \tilde{C}_{y i}^{-1}$.

Proof. Choose a stochastic Lyapunov function as

$$
V(S(k), k)=S^{T}(k) P_{i} S(k)
$$

for $\theta_{k}=i, \theta_{k+1}=j$, we could get

$$
\begin{aligned}
E(\Delta V(S(k), k))= & E(V(S(k+1))-V(S(k))) \\
& =S^{T}(k+1) Q_{i} S(k+1)-S^{T}(k) P_{i} S(k)(29)
\end{aligned}
$$

Substituting the sliding mode equation (11) and the control law (27) and (28)to the above equation, we could obtain

$$
\begin{gathered}
E(V(S(k), k))=\left[\tilde{G}_{i} \tilde{A}_{i} z(k)+\tilde{G}_{i} \tilde{B}_{i}[u(k)+F(u, x, k)]\right]^{T} Q_{i} \\
{\left[\tilde{G}_{i} \tilde{A}_{i} z(k)+\tilde{G}_{i} \tilde{B}_{i}[u(k)+F(u, x, k)]\right]-S^{T}(k) P_{i} S(k)}
\end{gathered}
$$

Substituting (27) and (28) to the above equation, it could be rewritten as

$$
\begin{aligned}
E(V(S(k), k))= & S^{T}(k)\left[(1-q T)^{2} Q_{i}-P_{i}\right] S(k)-2 \epsilon T(1-q T)\|S(k)\| Q_{i} \\
& +(\epsilon T)^{2}\|\operatorname{sign} S(k)\| Q_{i}\left[\tilde{G}_{i} \tilde{B}_{i}\left(F(u, x, k)+\bar{u}_{1}\right)\right] \\
& -2 \epsilon T \operatorname{signS}(k) Q_{i}\left[\tilde{G}_{i} \tilde{B}_{i}\left(F(u, x, k)+\bar{u}_{1}\right)\right] \\
& -2(1-q T)\left[\tilde{G}_{i} \tilde{B}_{i}\left(F(u, x, k)+\bar{u}_{1}\right)\right]^{T} Q_{i} S(k) \\
& +\left[\tilde{G}_{i} \tilde{B}_{i}\left(F(u, x, k)+\bar{u}_{1}\right)\right]^{T} Q_{i}\left[\tilde{G}_{i} \tilde{B}_{i}\left(F(u, x, k)+\bar{u}_{1}\right)\right]
\end{aligned}
$$

according to

$\left\|\bar{u}_{1}\right\|-\|F(u, x, k)\| \leq\left(F(u, x, k)+\bar{u}_{1}\right) \leq\|F(u, x, k)\|+\left\|\bar{u}_{1}\right\|$, we get $\left[\tilde{G}_{i} \tilde{B}_{i}\left(F(u, x, k)+\bar{u}_{1}\right)\right]^{T} Q_{i}\left[\tilde{G}_{i} \tilde{B}_{i}\left(F(u, x, k)+\bar{u}_{1}\right)\right] \leq\left\|\tilde{G}_{i} \tilde{B}_{i}\right\|^{2}(-\gamma)^{2} \| Q_{i}$

From the above equations, if $(1-q T)^{2} Q_{i}-P_{i}<0$ and $\gamma\left\|\tilde{G}_{i} \tilde{B}_{i}\right\|-2(1-q T)\|S(k)\|<0$ satisfied, then we could get $(29)<0$. Then the system (7) is stochastically stabilized.

\section{B. Dynamic output feedback control}

When the system does not satisfy the 'Kimura-Davison' condition, a dynamic output feedback control law in this section will be designed.

$$
\left\{\begin{array}{l}
x_{c}(k+1)=A_{c i} x_{c}(k)+B_{c i} y(k) \\
u(k)=C_{c i} x_{c}(k)+D_{c i} y(k)+\bar{u}_{2}
\end{array}\right.
$$


where $A_{c i}, B_{c i}, C_{c i}, D_{c i}$ are the matrices needed to be determined, and $\bar{u}_{2}$ is compensated for the uncertain part $F(u, x, k)$, it is defined as

$$
\bar{u}_{2}=-\frac{1}{1-\alpha}\left[\alpha\left\|C_{c i} x_{c}(k)+D_{c i} y(k)\right\|+\beta(y, k)\right] \frac{S}{\|S\|}
$$

The uncertain system (2) together with (37), then the new system could be written as following

$$
\left\{\begin{array}{l}
\tilde{x}(k+1)=\tilde{A} \hat{x} \tilde{x}(k)+\tilde{B}_{i}\left[\bar{u}_{2}+F(u, x, k)\right] \\
\tilde{y}(k)=\bar{C} \tilde{x}(k)
\end{array}\right.
$$

where

$$
\begin{gathered}
\tilde{x}=\left[\begin{array}{c}
x(k) \\
x_{c}(k)
\end{array}\right], \tilde{y}(k)=\left[\begin{array}{c}
y(k) \\
x_{c}(k)
\end{array}\right], \\
\tilde{A}_{i}=\bar{A}_{i}+\bar{B}_{i} K_{i} \bar{C}_{i}, \tilde{B}_{i}=\left[\begin{array}{c}
B_{i} \\
0
\end{array}\right], \bar{A}_{i}=\left[\begin{array}{cc}
A_{i} & 0 \\
0 & 0
\end{array}\right], \bar{B}_{i}=\left[\begin{array}{cc}
B_{i} & 0 \\
0 & I
\end{array}\right], \\
K_{i}=\left[\begin{array}{ll}
D_{c i} & C_{c i} \\
B_{c i} & A_{c i}
\end{array}\right], \bar{C}_{i}=\left[\begin{array}{cc}
C_{y i} & 0 \\
0 & I
\end{array}\right] .
\end{gathered}
$$

The sliding mode function is chosen as following:

$$
S=N \tilde{y}(k)=N \bar{C} \tilde{x}(k)=N_{1} C_{y i} x(k)+N_{2} x_{c}=N_{1} y(k)+N_{2} x_{c} .
$$

Lemma2 Let $M$ and $N$ be two real constant matrices of compatible dimensions, then the following equation holds

$$
\pm M^{T} N \pm N^{T} M \leq M^{T} M+N^{T} N
$$

Theorem 3 The closed-loop system (32) is stochastically stable if there exist a set of positive matrices $X_{i}, i \in \sigma$ and matrices $Y_{i}, \forall i \in \sigma$ such that the following LMI is satisfied

$$
\left[\begin{array}{ccc}
-X_{i} & * & * \\
\left(\bar{A}_{i} X_{i}+\bar{B}_{i} Y_{i} \bar{C}_{i}\right) \mathcal{L} & -X_{j} & 0 \\
\bar{A}_{i} X_{i}+\bar{B}_{i} Y_{i} \bar{C}_{i} & 0 & -I
\end{array}\right]<0
$$

where the $\pi_{i}, \chi_{j}$ are same to that defined in (20) and (21) and moreover if (35) holds, the parameter of the designed controller $K_{i}=Y_{i}\left(\bar{C} X_{i} \bar{C}^{-1}\right)^{-1}$.

Proof Choose a stochastic Lyapunov function as

$$
V(k)=\tilde{x}^{T}(k) P_{i} \tilde{x}(k), \quad \forall i \in \sigma
$$

Based on the Definition 1 and Lemma 1, define

$$
\begin{aligned}
E(\Delta V) & =E(V(\tilde{x}(k+1), j \mid \tilde{x}(k), i)-V(\tilde{x}(k), i) \\
& =\tilde{x}(k+1)^{T}\left(\sum_{j=1}^{N} p_{i j} P_{j}\right) \tilde{x}(k+1)-\tilde{x}(k)^{T} P_{i} \tilde{x}(k)
\end{aligned}
$$

Denote $Q_{i}=\sum_{j=1}^{N} p_{i j} P_{j}$, and according to system (32), the above equation could be written as

$$
\begin{aligned}
& =\tilde{x}^{T}(k)\left(\tilde{A}_{i}^{T} Q_{i} \tilde{A}_{i}-P_{i}\right) \tilde{x}(k)+\tilde{x}^{T}(k) \tilde{A}_{i}^{T} Q_{i} \tilde{B}_{i}\left[F(u, x, k)+\bar{u}_{2}\right] \\
& +\left\{\tilde{B}_{i}\left[F(u, x, k)+\bar{u}_{2}\right]\right\}^{T} Q_{i} \tilde{A} \tilde{x}_{i}(k)+\left\{\tilde{B}_{i}\left[F(u, x, k)+\bar{u}_{2}\right]\right\}^{T} Q_{i} \tilde{B}_{i}\left[F(u, x, k)+\bar{u}_{2}\right]
\end{aligned}
$$

According to Lemma2, we could obtain

$$
E(\Delta(V)) \leq\left[\begin{array}{c}
\tilde{x}(k) \\
F(u, x, k)+\bar{u}_{2}
\end{array}\right]^{T} \mathcal{G}_{i}\left[\begin{array}{c}
\tilde{x}(k) \\
F(u, x, k)+\bar{u}_{2}
\end{array}\right]
$$

where $\mathcal{G}_{i}=\left[\begin{array}{cc}\tilde{A}_{i}^{T} Q_{i} \tilde{A}_{i}-P_{i}+\tilde{A}_{i}^{T} \tilde{A}_{i} & 0 \\ 0 & \tilde{B}_{i}^{T} Q_{i} \tilde{B}_{i}+\tilde{B}_{i}^{T} Q_{i} Q_{i} \tilde{B}_{i}\end{array}\right]$

As $Q_{i}>0$, it is easy to obtain $\tilde{B}_{i}^{T} Q_{i} \tilde{B}_{i}+\tilde{B}_{i}^{T} Q_{i} Q_{i} \tilde{B}_{i}>0$ so if the item

$$
\tilde{A}_{i}^{T} Q_{i} \tilde{A}_{i}-P_{i}+\tilde{A}_{i}^{T} \tilde{A}_{i}<0,
$$

then $\mathcal{G}_{i}<0, E(\Delta(V))<0$, system (32) is stochastically stabilized.

Applying Schur complement to (37), we could obtain

$$
\left[\begin{array}{cccccc}
-P_{i} & \tilde{A}_{i}^{T} \sqrt{p_{i 1}} & \tilde{A}_{i}^{T} \sqrt{p_{i 2}} & \cdots & \tilde{A}_{i}^{T} \sqrt{p_{i N}} & \tilde{A}_{i}^{T} \\
* & -P_{1}^{-1} & 0 & 0 & 0 & 0 \\
* & * & -P_{2}^{-1} & 0 & 0 & 0 \\
* & * & * & \ddots & 0 & 0 \\
* & * & * & * & -P_{N}^{-1} & 0 \\
* & * & * & * & * & -I
\end{array}\right]<0
$$

Pre- and post- multiply $\operatorname{diag}\left\{P_{i}^{-1}, I, I, \cdots, I, I\right\}$ to (38) and let $X_{i}=P_{i}^{-1}, \mathcal{L}=\left[\begin{array}{llll}\sqrt{p_{i 1}} & \sqrt{p_{i 2}} & \cdots & \sqrt{p_{i N}}\end{array}\right]$, substituting $\tilde{A}_{i}=\bar{A}_{i}+\bar{B}_{i} K_{i} \bar{C}_{i}$ to (38), we could get

$$
\left[\begin{array}{ccc}
-X_{i} & \left(X_{i} \bar{A}_{i}^{T}+\bar{C}_{i}^{T} Y_{i}^{T} \bar{B}_{i}^{T}\right) \mathcal{L} & X_{i} \bar{A}_{i}^{T}+\bar{C}_{i}^{T} Y_{i}^{T} \bar{B}_{i}^{T} \\
* & -X_{j} & 0 \\
* & * & -I
\end{array}\right]<0
$$

Therefore, if there exist post symmetric matrix $X_{i}$ and matrix $Y_{i}$, guarantee that the inequality (39) holds, we could 
easily get $\mathcal{G}_{i}<0$. And then(35) holds, the closed system (32) is stochastically stable. This completes the proof. Moreover the desired controller parameters could be calculated by

$$
K_{i}=Y_{i} \bar{C}_{i} X_{i}^{-1} \bar{C}_{i}^{-1}
$$

\section{CONCLUSION}

In this paper, sliding mode feedback control problem of discrete-time MJS with uncertainties has been discussed. Static and dynamic output feedback control strategies are proposed. Some sufficient conditions in terms of LMIs, under which the MJS could be stochastic stabilized by the constructed controllers, have been presented.

\section{ACKNOWLEDGMENT}

This work is supported by Key scientific research projects in Universities of Henan Province \#16A120001 and China National Natural Science Foundation \\#61403124.

\section{REFERENCES}

[1] N. N. Krasovskii, E A.Lidskii, "Analytical design of controllers in systems with random attribute," Automation and Remote Control, vol.22, no.9, pp.1021-2025, 1961.

[2] C.E. de Souza, M.D. Fragoso, "H $\mathrm{H}_{\infty}$ control for linear systems with Markovian jumping parameters ," Control-Theory and Advanced Technology, vol.9, no.2, pp. 457-466, 1993.

[3] Z. D. Wang, H. Qiao and K. J. Burnham, "On Stabilization of bilinear uncertain time-Delay stochastic systems with Markovian jumping parameters," IEEE Transactions on Automatic Control, vol. 47, no. 4, pp. 640-646,2002.

[4] L. Li and V.A. Ugrinovskii, "On necessary and sufficient conditions for $\mathrm{H}_{\infty}$ output feedback control of Markov jump linear systems," IEEE Transactions on Automatic Control, vol. 52, no.7, pp.1287-1292, 2007.

[5] C.E. de Souza, "Robust stability and stabilization of uncertain discretetime jump linear systems," IEEE Transactions on Automatic Control, vol. 51, no. 5, pp. 836-841, 2006.

[6] P. Shi, Y. Xia, G. P. Liu and D. Rees, "On designing of sliding-mode control for stochastic jump systems," IEEE Transactions on Automatic Contrl, vol. 51, no. 1, pp. 97-103, 2006.

[7] L. G. Wu and D. W. C. Ho, "Sliding mode control of singular stochastic hybrid systems," Automatica, vol. 46, no. 4, pp.779-783, 2010.

[8] O. L. V. Costa, M. D. Fragoso, and R. P. Marques, "Discrete-time Markovian jump linear systems," London: Springer-Verlag, 2005.

[9] de Souza, C. E., A. Trofino and K. A. Barbosa, "Mode-independent $\mathrm{H}_{\infty}$ filters for Markovian jump linear systems," IEEE Transactions on Automatic Control, vol. 51, no. 11, pp. 1837-1841, 2006.

[10] P. Shi, Y. Xia, G. P. Liu and D. Rees, "On designing of sliding-mode control for stochastic jump systems," IEEE Transactions on Automatic Contrl, vol. 51, no. 1, pp. 97-103, 2006. 